Agriculture suisse et mondialisation

\title{
Le monde paysan suisse face à l'ouverture des marchés. Craintes et réactions
}

\section{Gérard Vuffray}

\section{(2) OpenEdition}

1 Journals

Édition électronique

URL : http://journals.openedition.org/aspd/921

DOI : 10.4000/aspd.921

ISSN : 1663-9669

Éditeur

Institut de hautes études internationales et du développement

\section{Édition imprimée}

Date de publication : 1 avril 2002

Pagination : 27-38

ISSN : 1660-5934

\section{Référence électronique}

Gérard Vuffray, "Le monde paysan suisse face à l'ouverture des marchés. Craintes et réactions »,

Annuaire suisse de politique de développement [En ligne], 21 | 2002, mis en ligne le 06 septembre 2012 consulté le 08 septembre 2020. URL : http://journals.openedition.org/aspd/921 ; DOI : https://doi.org/ 10.4000/aspd.921 


\title{
LE MONDE PAYSAN SUISSE FACE À L'OUVERTURE DES MARCHÉS. CRAINTES ET RÉACTIONS
}

\author{
GÉRARD VUFFRAY*
}

\section{LA SUISSE: UN DES MARCHÉS LES PLUS OUVERTS AU MONDE EN MATIËRE DE PRODUITS AGRICOLES ET ALIMENTAIRES}

Avec un taux qui oscille entre $58 \%$ et $62 \%$ de ses besoins selon les années, la Suisse figure parmi les pays dont le taux d'autoapprovisionnement alimentaire est le plus bas de la planète, et dont le marché est en corollaire parmi un des plus ouverts qui soient en termes d'importations de produits agricoles et alimentaires. Il est essentiel de rappeler en préambule ce paramètre fondamental, qui va à l'encontre de beaucoup d'idées reçues et dont on ne tient généralement aucun compte dans les débats sur l'évolution future de l'ouverture des marchés, que ce soit dans le cadre de négociations bilatérales avec différents pays, dans le cadre des négociations au sein de l'OMC ou encore lorsqu'on parle de libéralisation totale des importations agricoles en provenance des pays les plus pauvres.

C'est donc en gardant toujours à l'esprit cette donnée essentielle qu'il faut aborder la question, tout en sachant qu'il n'y aurait vraisemblablement pas beaucoup d'autres pays qui seraient prêts à accepter un tel taux moyen d'importations de $40 \%$ de leurs besoins alimentaires, même parmi les plus ardents promoteurs actuels du libre-échange en la matière, que ce soient les Etats-Unis, l'Union européenne ou les pays du groupe de Cairns. Le taux d'autoapprovisionnement de la Suisse varie de $18 \%$ pour les graisses et huiles végétales à $111 \%$ pour les produits laitiers, en passant par $52 \%$ pour les légumes, $62 \%$ pour les céréales et $70 \%$ pour l'ensemble des viandes ${ }^{1}$.

\section{LES CONDITIONS-CADRES PARTICULIÈRES DE L'AGRICULTURE SUISSE}

L'agriculture suisse dans son ensemble est soumise en outre à au moins deux types de contraintes fort distinctes de celles de la plupart des pays. Et c'est également en gardant en tête l'existence de ces contraintes que la question de l'ouverture du marché aux produits importés doit être examinée:

口 il s'agit d'une agriculture de montagne, plus de la moitié des surfaces utilisées se situant à une altitude supérieure à $1000 \mathrm{~m}$. La topographie y est tourmentée, les conditions climatiques rudes; les exigences en matière d'environnement, de méthodes de production et de détention des animaux y sont en moyenne beaucoup plus sévères et contrôlées qu'ailleurs;

口 l'agriculture suisse est entièrement intégrée au contexte économique suisse. Ses coûts de production sont intimement liés à ceux des facteurs de

* Ingénieur agronome, secrétaire d'Uniterre.

1. Office fédéral de l'agriculture, Rapport agricole 2000 et Rapport agricole 2001, Berne. 
production suisses, qui sont parmi les plus élevés du monde: coûts de la terre, des constructions et des machines, des services et de la main-d'œuvre, des transports et de l'énergie, des engrais, des assurances, etc. ${ }^{2}$ Le prix de vente de ses produits dépend donc directement du niveau des coûts de l'environnement économique et social suisse.

En résumé, tous ces facteurs renchérissent de manière importante les coûts des produits agricoles du pays, coûts qui se répercutent ensuite à travers des filières de transformation et de distribution jusqu'au consommateur. Les prix des produits agricoles du pays resteront toujours très supérieurs à ceux des produits de nombreux autres pays, quelles que soient la compétence et la productivité des paysans suisses.

Voilà qui pose, certes de manière simplifiée, l'équation initiale du problème auquel sont confrontés les milieux paysans suisses lorsqu'on aborde la question d'une ouverture encore plus grande des frontières. Mais il faut y ajouter un autre élément, qui est celui du mandat de service public que l'agriculture helvétique remplit depuis quelques années.

\section{MULTIFONCTIONNALITÉ ET DÉVELOPPEMENT DURABLE}

L'agriculture a été l'objet de débats démocratiques intenses pendant plus d'une dizaine d'années en Suisse, avec à la clé plusieurs votations populaires fédérales, la modification de la Constitution, l'adoption d'une nouvelle loi sur l'agriculture et la refonte presque complète de l'ensemble des lois et ordonnances qui s'y rapportent. Le rôle de l'agriculture et le mode de rémunération des paysans ont subi des changements fondamentaux. L'économie de marché ne veut pas prendre en compte dans le prix et la valeur des produits agricoles les coûts environnementaux, sociaux et culturels qui leur sont liés. Elle a en conséquence imposé le découplage des prix agricoles en deux parties, l'une à la charge de la collectivité, l'autre payée par le marché. C'est notamment sous la pression du GATT et des négociations qui se sont déroulées au niveau international entre 1989 et 1995 que les notions de multifonctionnalité et de développement durable ont été précisées et détachées des valeurs marchandes monnayables sur les marchés des produits agricoles.

Selon le nouvel article 104 de la Constitution fédérale, l'agriculture est devenue un acteur central, d'une part, de la conservation des ressources naturelles et de l'entretien du paysage rural, et, d'autre part, du maintien d'une population et d'une gestion du territoire en zones rurales, c'est-à-dire sur près de la moitié du territoire national. Elle conserve en outre son mandat prioritaire, qui est aussi jugé de nature «non commerciale», à savoir contribuer à la sécurité alimentaire du pays. Les prestations dites «non commerciales» de l'agriculture sont désormais rémunérées par des paiements directs versés par la Confédération aux paysans. Elles représentent un catalogue complexe d'exigences et de contraintes à fournir, sous forme contractuelle et volontaire, et sous contrôle permanent des Cantons. Elles sont en principe totalement découplées de la valeur marchande des produits alimentaires vendus sur le marché.

2. Union suisse des paysans, Possibilités de réduire les coûts de production dans l'agriculture, 1996. 
La nouvelle approche du rôle de l'agriculture a abouti en fait à la création d'un double marché et d'une double clientèle: d'un côté les consommateurs achètent des produits alimentaires, de l'autre côté la Confédération achète des services, parmi lesquels figurent la sécurité alimentaire du pays, la conservation de ses ressources naturelles, l'occupation décentralisée du territoire et l'entretien de son paysage rural. Sur les deux marchés, produits alimentaires et services, le travail et les prestations fournis par l'agriculture ont un prix, qui est conditionné par le niveau de vie suisse, par les coûts de production suisses, le niveau des exigences des deux clientèles, etc.

Les objectifs à atteindre en matière d'occupation du territoire rural, d'entretien du paysage et de conservation des ressources naturelles sont souvent difficiles à définir et à traduire dans des mesures et dans des contrats applicables sur des périodes de temps durables et garanties. Inutile de préciser qu'on peut longuement épiloguer sur la valeur économique de chaque prestation «non commerciale». Quel est le juste prix de $7 \%$ à $10 \%$ de surfaces de compensation écologique, soustraites à la production alimentaire pour préserver la biodiversité en zones rurales, sauvegarder la flore et la faune? Quel est le juste prix à payer pour sauvegarder certains paysages et ramener certains milieux plus proches de l'état naturel? Quel est le prix à payer pour des œufs de poules en liberté, pour des viandes sans hormones, sans utilisation d'antibiotiques ou sans organismes génétiquement modifiés, ou pour tout simplement assurer le maintien d'une agriculture dans le pays?

\section{LA QUADRATURE DU CERCLE}

L'agriculture suisse est aujourd'hui confrontée à un défi qui relève de la quadrature du cercle: on lui demande de poursuivre des objectifs constitutionnels quasiment contradictoires, à savoir plus de compétitivité et plus d'écologie, travailler plus rationnellement, à moindres coûts, avec des prix et des revenus agricoles à la baisse, des contraintes écologiques à la hausse. Plus de 20’000 exploitations ont disparu en dix ans $(25 \%)$, avec un rythme de disparitions qui va en s'accélérant. Le revenu moyen des paysans restants stagne pourtant toujours autour de 3000 francs par mois, paiements directs inclus, soit selon les régions de $35 \%$ à $55 \%$ de moins que pour les catégories comparables de personnes actives dans les autres secteurs de l'économie ${ }^{3}$.

C'est donc dire que des pressions constantes pour une ouverture encore plus large aux importations ne sont généralement pas bienvenues dans le monde paysan, d'autant plus qu'une grande partie des produits importés ne satisfont pas aux exigences environnementales et de qualité imposées aux produits suisses. D'autre part, il n'y a aucun doute que la plupart des produits alimentaires importés peuvent arriver aux frontières à une fraction du coût de production helvétique, et que sans une protection adéquate il n'y a pas d'avenir possible pour une production alimentaire en Suisse.

3. FAT (Station fédérale de recherches en économie et technologie agricoles - Taenikon), Evolution économique de l'agriculture suisse de 1990 à 1999, Rapports FAT, nº 556, 2000. 


\section{LA GRANDE DÉVALORISATION DU TRAVAIL DE LA TERRE}

Tout citoyen moyen semble avoir intégré l'idée qu'il est normal d'acheter des produits alimentaires importés au plus bas prix possible, ce qui aboutit de facto à une pression permanente à la baisse sur le salaire perçu par les paysans pour leur travail. Mais le même citoyen s'opposerait sans aucun doute farouchement, et de manière justifiée, à son remplacement dans sa place de travail par une personne venue d'Afrique ou d'Asie, et payée, à compétences égales, au tarif de son pays d'origine. Pourquoi y a-t-il une telle différence de perception du problème? Mystère et paradoxe! Et pourtant les deux cas de figure sont parfaitement comparables...

Autre évolution significative, la part des dépenses alimentaires dans le budget global des ménages suisses n'a cessé de décroître au fil des années: de $27 \%$ en 1960, elle est descendue en dessous de $10 \%$ dans les années 1990, et se situe aujourd'hui à $7 \%$. Entre 1988 et 1998, la facture alimentaire des consommateurs suisses a bien augmenté en valeur absolue de 23 à 27 milliards de francs, mais dans le même laps de temps, la part revenant aux paysans a chuté de $38 \%$ à $24 \%$, tandis que celle des intermédiaires et de la distribution est passée de $62 \%$ à $76 \%$. Les importants gains de productivité obtenus par l'agriculture pendant cette période ont été entièrement absorbés par les filières agroalimentaires situées en amont et en aval de l'agriculture. Une meilleure répartition de la valeur ajoutée de ce secteur économique et un renchérissement du coût de l'alimentation s'avéreront indispensables à l'avenir, si l'on admet que les paysans, mais aussi d'autres catégories de personnes de la filière agroalimentaire, comme les ouvriers agricoles ou les employés de la grande distribution, doivent gagner un revenu décent pour leur travail ${ }^{4}$.

\section{LA PRESSION IDÉOLOGIQUE INTERNE}

Les orientations récentes de la politique agricole de l'administration fédérale sont très inquiétantes. Un projet de révision de la Loi sur l'agriculture et du droit foncier rural, intitulé «Politique agricole 2007» (PA 2007), est en cours d'élaboration: il est entièrement dominé par un seul objectif, à savoir la fourniture au plus bas prix de matières premières aux industries agroalimentaires et à la distribution. Pour y arriver, une stratégie, l'accélération programmée de la restructuration de l'agriculture, c'est-à-dire l'élimination le plus rapidement possible de $30 \%$ à $40 \%$ des exploitations agricoles du pays, qui sont jugées non compétitives et donc non viables à long terme.

Tel que proposé, le projet PA 2007 constitue une réinterprétation idéologique du consensus constitutionnel atteint en 1998 après plus de dix ans de débats démocratiques et de votations fédérales, et la remise en question du mandat confié à l'agriculture, en voulant privilégier à tout prix l'objectif de compétitivité au détriment de tous les autres. Les auteurs du projet ont énormément de peine à dissimuler qu'ils n'ont pas vraiment digéré la complexité et la multiplicité des tâches attribuées à l'agriculture par l'article 104 de la Constitution ${ }^{5}$. Le

4. Office fédéral de l'agriculture, Rapport agricole 2000 et Rapport agricole 2001, Berne.

5. Voir en annexe l'article 104 de la Constitution fédérale. 
lancement de cette nouvelle réforme de la politique agricole, trois ans à peine après l'entrée en vigueur de la réforme précédente «Politique agricole 2002 », est une marque de grande précipitation dont dépend le sort de dizaines de milliers de familles paysannes. Aucune échéance internationale ne l'impose; il s'agit d'un programme d' «ajustement structurel» et de démantèlement à cent pour cent d'origine suisse.

\section{UNE AUTRE VISION DE L'AVENIR}

L'avenir de l'agriculture suisse ne réside ni dans l'élimination de $30 \%$ à $40 \%$ des paysans, ni dans la fourniture de quantités non maîtrisées de matières premières à vils prix. Il réside dans le maintien du plus grand nombre possible d'exploitations familiales sur tout le territoire, dans la promotion d'une agriculture de proximité et de la gestion de l'offre (maîtrise des quantités), dans des produits de qualité haut de gamme, dans l'obtention de prix rémunérateurs correspondant au niveau de vie du pays, dans le développement de nouveaux modes d'organisation et de collaboration entre producteurs, dans le rééquilibrage des rapports de force entre partenaires commerciaux sur les marchés, dans le développement de relations plus étroites entre paysans et citoyens consommateurs, dans la fourniture d'une large palette de prestations multifonctionnelles à la collectivité ainsi que dans le désendettement des exploitations.

\section{LA PRESSION DE L'OMC}

Les craintes et les revendications vis-à-vis de l'OMC ne sont pas forcément uniformes et identiques dans tous les milieux paysans de Suisse. Mais il y a unanimité sur le fond, à savoir que les dimensions sociales, environnementales, éthiques, culturelles et de sécurité alimentaire doivent être traitées en priorité avant toute nouvelle libéralisation dans le domaine de l'agriculture. Il y a donc une opposition formelle largement partagée à de nouvelles négociations à l'OMC, si elles ont pour objectif d'aboutir à de nouvelles libéralisations, tant que les autres questions n'auront pas été traitées de manière prioritaire et satisfaisante.

En ce qui concerne le mouvement Uniterre, syndicat paysan de Suisse romande membre de la Coordination paysanne européenne et de la Via Campesina sur le plan international, l'opposition au nouveau cycle de négociations est basée sur des considérations plus larges, puisque nous demandons à la fois la reconnaissance explicite de la multifonctionnalité et de la durabilité de l'agriculture, ainsi que celle de la souveraineté alimentaire des pays. Cette souveraineté alimentaire est un droit légitime des autorités publiques des pays de décider la politique de sécurité alimentaire qu'ils entendent mener pour leur propre population, y compris si nécessaire au moyen de soutiens internes accrus de leur agriculture et de protections renforcées aux frontières. Nous nous opposons également à la privatisation du vivant par le biais des brevets, ainsi qu'à l'élargissement des compétences de l'OMC notamment dans le domaine des services publics. L'accès à l'eau, aux semences, aux services de santé, à l'éducation, etc., doit rester un droit universel humain; ces services ne doivent pas devenir accessibles qu'aux personnes ayant les moyens financiers de se les payer. 
Nous demandons également que des réformes profondes de l'institution OMC et de ses mécanismes de décision soient entreprises avant d'envisager d'autres dérégulations globales: nous avons en particulier suggéré que l'OMC soit intégrée au sein des organisations spécialisées de l'ONU, que ses domaines de compétence soient redéfinis, que les règles commerciales soient intégrées à l'ensemble des dispositions de droit international. Dans la foulée, la création d'un tribunal du commerce international indépendant devrait remplacer dans les plus brefs délais les procédures actuelles scandaleuses de règlement des différends entre pays membres de l'OMC. Ces procédures autorisent aujourd'hui encore l'application de sanctions économiques collectives à des groupes de personnes, en particulier des paysans, qui n'ont strictement rien à voir avec les différends en cause, et où les instances de l'OMC y sont à la fois juge et partie.

\section{L'AGRICULTURE EN POSITION DE FAIBLESSE}

Mais il y a une seconde catégorie de préoccupations pour l'agriculture suisse, qui ressort lors de chaque négociation internationale, que ce soit au niveau bilatéral ou au niveau de l'OMC. La part de l'agriculture dans la valeur ajoutée de l'économie suisse est devenue au fil des ans de plus en plus faible, pour atteindre à peine $1,1 \%$ du total à la fin 1998 (4 milliards de francs sur 377 milliards). Si on y ajoute les prestations de service rendues à la collectivité, évaluées à environ 2 milliards de francs par an, le poids de l'agriculture atteint à peine $1,5 \%$ de celui de l'économie suisse. Au niveau du commerce extérieur, les importations de produits agricoles représentent en 1999 6,8\% des importations totales du pays ( 8 milliards sur 120 milliards) et les exportations $2,7 \%$ (3,3 milliards sur 121 milliards $)^{6}$.

Est-il besoin de préciser que les intérêts de l'agriculture ne sont objectivement pas prioritaires dans le cadre de négociations commerciales internationales engagées par la Suisse? La crainte est réelle de les voir sacrifiés au profit d'autres branches plus puissantes de l'économie, d'autant plus qu'il s'agit à peu près du seul domaine où la Suisse ne pousse pas activement à plus de libéralisme.

Des concessions supplémentaires futures en matière d'ouverture des frontières (réduction des taxes à l'importation et augmentation des contingents tarifaires) et de réduction des soutiens internes à l'agriculture seront très mal accueillies par les milieux paysans. Il en va réellement de la survie de l'agriculture du pays, qui perd déjà $3 \%$ à $4 \%$ de ses exploitations chaque année. Il n'en reste aujourd'hui qu'environ 70'000 en activité. Par contre, l'abandon à terme des subventions à l'exportation semble légitime, dans tous les cas où elles peuvent être assimilées à des pratiques de dumping sur les marchés d'exportation. Pour la Suisse, ces subventions concernent au premier chef les exportations de fromages à pâte dure, essentiellement à destination de l'Union européenne. Elles seront réduites en cinq ans de plus de $75 \%$, dans le cadre des accords bilatéraux passés entre la Suisse et l'UE. 


\section{LIBÉRALISATION TOTALE DES IMPORTATIONS AGRICOLES POUR LES PMA?}

La Confédération propose de supprimer progressivement toutes les taxes à l'importation pour les produits agricoles en provenance du groupe des 49 pays les plus pauvres de la planète (PMA: pays les moins avancés), à la suite d'ailleurs d'une initiative similaire de l'Union européenne intitulée «tout sauf les armes». Les taxes seraient réduites en moyenne et dans un premier temps de $30 \%$ au $1^{\text {er }}$ janvier 2002, puis encore de $30 \%$ au début 2004 et enfin totalement supprimées en 2007. Les principaux arguments avancés en faveur de cette mesure sont bien sûr que les pays concernés vont pouvoir en tirer des bénéfices, qu'ils demandaient depuis longtemps ce geste de la part des pays riches, que des engagements avaient été pris dans ce sens à l'OMC, et que ce serait faire preuve d'égoïsme pur et simple que de ne pas y être favorable.

Le problème étant posé de cette manière, il apparaît au premier abord difficile et malvenu de s'opposer à une mesure qui met en avant la solidarité et le rééquilibrage des rapports internationaux entre pays riches et pays les plus pauvres. Mais dès qu'on creuse un tant soit peu la question, et que l'on cherche à savoir précisément quels pourraient être les effets au Nord comme au Sud d'un accès libre de notre marché pour tous les produits agricoles des PMA, la question prend une tout autre physionomie. La libéralisation telle que proposée ressemble à s'y méprendre à un traitement médical inapproprié, voire nocif, dont on augmente systématiquement les doses, en dépit des preuves accumulées de son incapacité à résoudre le mal qu'il est supposé soigner...

\section{LES PAYSANS SERONT LES PERDANTS DANS LES PMA...}

Absolument rien ne prouve que les pays les plus pauvres tireront un bénéfice réel de cet accès libre au marché agricole suisse, et surtout rien ne prouve que cet éventuel bénéfice profitera à leurs paysans : la Suisse ne prend en effet absolument aucun engagement pour garantir que les futures importations soient basées sur des prix rémunérateurs et équitables, et qu'elles puissent effectivement contribuer à l'amélioration du niveau de vie des populations qui en dépendent. Autrement dit, ce sont les prix mondiaux qui seront appliqués. La mesure n'a strictement plus rien de solidaire. Il s'agit tout au plus pour la Suisse de redorer son blason bien terni, d'améliorer son image à peu de frais (les millions de droits de douane non perçus), tout en faisant porter les conséquences économiques de la libéralisation par les paysans de là-bas et d'ici, qui seront tous perdants dans l'aventure.

Inciter les agricultures des pays les plus pauvres à produire pour l'exportation aux prix mondiaux vers la Suisse reviendrait à reproduire le mécanisme maintes fois dénoncé des exportations en sens inverse des excédents agricoles des pays riches, qui ont provoqué des ravages sur les agricultures vivrières de ces mêmes pays les plus pauvres. Selon toute vraisemblance, les seuls gagnants pourraient bien être les entreprises agro-industrielles d'envergure internationale profitant de la disparition des taxes pour s'implanter dans des PMA, accaparer des terres, employer de la main-d'œuvre à très bas prix et s'emparer de nouveaux marchés au détriment des paysans du Sud comme du Nord. 
Les paysans du Sud eux-mêmes ne s'y sont pas trompés: ils revendiquent avant tout le développement prioritaire de leurs propres marchés intérieurs, la protection de leur agriculture contre le dumping des surplus occidentaux à l'importation, l'accès à la terre, à l'eau, aux semences, la suppression de la clause d'accès au marché obligatoire de 5\% contenue dans l'Accord sur l'agriculture de l'OMC. La démarche proposée par la Suisse ne répond ni aux besoins ni aux demandes des paysans du Sud? ${ }^{7}$.

\section{... ET EN SUISSE}

Si d'autres conditions ne sont pas associées à l'accès au marché suisse pour des produits agricoles en provenance des 49 pays les plus pauvres, la suppression pure et simple des taxes à l'importation n'est pas acceptable: elle pourrait représenter une concurrence singulièrement déloyale pour un ou plusieurs produits agricoles majeurs qui constituent la base du revenu paysan en Suisse (par exemple le sucre), et exercer une pression particulièrement malvenue sur le revenu paysan suisse. Bien sûr, une clause de sauvegarde serait mise en place pour éviter de trop grands dérapages, mais elle n'ôterait pas la pression permanente à la baisse des prix par des produits arrivant aux frontières au niveau des prix mondiaux. Et on peut en outre supposer qu'on n'en restera pas là, et que le libre accès au marché suisse pourrait être ensuite étendu dans une prochaine étape à d'autres groupements de pays moins pauvres que les plus pauvres.

\section{PRODUITS TROPICAUX: LE LIBRE ACCÈS EST PARFAITEMENT JUSTIFIÉ}

D'un autre côté, la Suisse accorde déjà le libre accès aux PMA pour la quasitotalité des produits tropicaux, reconnaissant a priori leur vocation exportatrice naturelle vers les pays des zones climatiques tempérées. C'est parfaitement justifié, et cela ne met en péril ni nos produits ni notre agriculture, même si par exemple la présence simultanée sur nos étalages, pendant certaines périodes de l'année, de fruits tropicaux trop bon marché (paysans et ouvriers agricoles mal rémunérés) et de fruits indigènes peut nuire à l'écoulement de ces derniers.

\section{QUELS SONT LES PAYS CONCERNÉS?}

Parmi les 49 pays concernés classés parmi les plus pauvres de la planète, on trouve avant tout une grande partie de l'Afrique, avec 34 pays, sept pays d'Asie et diverses îles dispersées autour des mers du globe (dont Haïti).

La totalité des importations actuelles de la Suisse en provenance de ces 49 pays se monte en 1999 à 150 millions de francs, dont les deux tiers en provenance de quatre pays (Bangladesh, Liberia, Ouganda et Népal), et dont le tiers en produits agricoles. Les produits typiquement tropicaux tels que le café, le thé et les épices, qui bénéficient déjà d'une entrée libre en Suisse, ainsi que le tabac,

7. Voir en annexe la déclaration de Via Campesina et du ROPPA faite à propos d'une initiative similaire engagée par l'Union européenne. 
représentent plus de $80 \%$ des 50 millions de francs d'importations. Tabac mis à part (dont les importations ne semblent d'ailleurs pas souffrir des droits de douane existants), la libéralisation toucherait donc tous les autres produits, y compris le lait et les produits laitiers, les viandes, le sucre, les céréales et les huiles végétales ou animales. Elle ne porterait au point de départ que sur des marchandises d'une valeur d'environ 10 millions de francs, représentant au maximum quelques millions de francs de droits de douane.

\section{L'EXEMPLE DU SUCRE}

La menace paraît au premier abord très mince pour l'agriculture suisse, mais elle pourrait vite prendre de l'ampleur. Prenons l'exemple du sucre. Sa consommation mondiale ne change que très lentement d'une année à l'autre en fonction de l'évolution de la population, et les prix sur le marché mondial sont particulièrement instables et en moyenne inférieurs aux coûts de production de quasiment tous les pays du monde.

Le marché suisse est caractérisé par une gestion de l'offre. La production indigène est contingentée. Elle couvre, avec en gros 200'000 tonnes de sucre, $65 \%$ de la consommation du pays, les prix étant négociés directement entre les sucreries et les organisations de producteurs de betterave. Il s'agit donc d'un marché régulé et protégé des fluctuations et des avatars du marché mondial.

Parmi les 49 PMA, au moins neuf sont exportateurs de sucre (Birmanie, Congo, Laos, Madagascar, Malawi, Mozambique, Soudan, Tanzanie et Zambie), les quantités globales exportées étant relativement faibles, aux alentours de 350 '000 tonnes par an. Des raffineries y sont présentes qui pourraient traiter d'importantes quantités de sucre supplémentaires. Ouvrir la frontière sans limitation de quantités et sans taxes à du sucre en provenance des 49 PMA au prix mondial pourrait rapidement se répercuter par l'effondrement du marché interne en l'absence de dispositions appropriées allant bien au-delà de l'utilisation toujours problématique et temporaire d'une clause de sauvegarde. Il faut en conséquence envisager d'autres mesures si l'on veut à la fois prétendre montrer de la solidarité vis-à-vis des PMA et assurer un revenu décent aux producteurs de betterave suisses. La solution réside dans l'octroi d'un contingent d'importation à des prix rémunérateurs et garantis aux PMA, quota qui serait pris sur les importations actuelles de plus de 100'000 tonnes de sucre par an, et qui pourrait être périodiquement révisé à la hausse. Les conditions seraient alors réunies pour que les paysans des PMA et de la Suisse soient gagnants dans une opération de commerce équitable.

\section{POUR UNE VÉRITABLE SOLIDARITÉ AVEC LES PAYSANS DES PMA}

La liberté d'accès au marché suisse pour les produits agricoles des PMA n'entrant pas en concurrence directe avec des produits de l'agriculture indigène est déjà très largement acquise. Si l'on veut étendre cette liberté d'accès aux produits qui sont aussi obtenus par l'agriculture suisse, les règles suivantes devraient s'appliquer: 
a attribution de quotas d'importation à prix garantis aux 49 pays les plus pauvres, les quotas étant pris sur les importations existantes;

- mise en place d'un système de restitution des droits de douane aux PMA plutôt que leur suppression, avec l'affectation des sommes restituées prioritairement à la rémunération des paysans, à l'amélioration des infrastructures agricoles ou au désendettement des PMA;

a reconnaissance en contrepartie par les PMA des objectifs multifonctionnels de l'agriculture dans le cadre des négociations à l'OMC, et acceptation de livrer des produits qui soient conformes aux exigences minimales de production devant être respectées par l'agriculture suisse (environnementales, phytosanitaires, etc.) ;

- exigence de traçabilité et de transparence sans compromis concernant la provenance exacte des produits importés, pour éviter des fraudes et des trafics triangulaires entre des pays tiers et les PMA. La tentation de déclarer faussement en provenance des PMA des produits agricoles d'autres pays pourrait en effet se révéler irrésistible afin de contourner le paiement des taxes à l'importation en Suisse;

- réflexions à mener sur le bien-fondé ou non d'encourager l'exportation de produits animaux, grands consommateurs de calories et de fourrages dont les populations des PMA devraient bénéficier en priorité pour leur propre alimentation. 


\section{Annexe 1: Déclaration de Via Campesina et du ROPPA}

La Via Campesina et le Réseau des organisations paysannes et de producteurs agricoles de l'Afrique de I'Ouest (ROPPA) dénoncent la libéralisation des échanges de produits agricoles prônée par l'OMC, et les politiques de dumping agricole pratiquées par les grandes puissances exportatrices vis-à-vis des pays tiers - en particulier les pays les moins avancés (PMA) - qui ruinent les capacités productives et les habitudes alimentaires tout en faisant disparaître I'agriculture paysanne du Nord et du Sud. Par exemple, I'exportation des excédents européens vers les PMA, à des prix très inférieurs aux prix pratiqués en Europe et dans les PMA, a ruiné la production agricole de ces pays.

La décision de l'Union européenne d'ouvrir sans droits de douane son marché agricole aux produits des PMA est le contraire d'une solution pour ces pays. Elle est faite pour justifier la pénétration des marchés des PMA par les exportateurs de I'UE plutôt que pour donner une réelle chance aux agriculteurs des PMA de vendre leurs productions en Europe.

Pour la Via Campesina et le Réseau des organisations paysannes et de producteurs agricoles de I'Afrique de l'Ouest, la priorité doit être donnée à une production vivrière, saine, de bonne qualité, culturellement appropriée, pour le marché intérieur de chaque pays et pour le marché sous-régional ou régional de chaque région du monde.

Les priorités des paysans et de leurs familles dans les PMA sont d'abord de pouvoir produire pour leur famille, puis d'avoir accès au marché intérieur, bien avant d'exporter. La décision européenne ne va au contraire que renforcer les bénéfices des grandes firmes utilisant les ressources et la main-d'œuvre des pays les moins avancés pour les cultures d'exportation vers l'UE. Cela diminuera les ressources et la main-d'œuvre consacrées à la production d'aliments pour les familles paysannes et urbaines de chaque pays, augmentant ainsi l'insécurité alimentaire.

Via Campesina et le ROPPA réaffirment:

- le droit pour les populations et leurs Etats à définir leurs politiques agricoles et alimentaires au niveau national, puis au niveau régional, au service des exploitations familiales agricoles, à condition que ces politiques soient sans dumping vis-à-vis des pays tiers: c'est la souveraineté alimentaire;

- le droit d'accès aux ressources (terre, eau, semences) afin de pouvoir produire, de pouvoir vivre dignement, voilà le problème des paysans des pays du Sud, auxquels ces droits aujourd'hui encore sont niés;

- le droit pour les pays/groupes de pays du Sud et du Nord à protéger leurs agricultures et leurs marchés afin de rémunérer équitablement le travail et les produits des exploitations familiales agricoles;

- le marché mondial ne doit plus être un marché de surplus mais un marché d'échanges équitables entre produits régionaux à des prix rémunérateurs. Les marchés internationaux doivent être régulés afin de stopper la détérioration des termes de l'échange, en particulier pour les paysans du Sud exportant des produits tropicaux.

Via Campesina et le ROPPA demandent aux gouvernements du Nord comme du Sud:

- de mettre en place ou de réformer des politiques agricoles pour qu'elles assurent la viabilité des exploitations familiales agricoles;

- de s'abstenir d'apporter des appuis et des ressources de la coopération internationale conditionnés par des obligations de libéralisation et de privatisation non conformes aux priorités des pays;

• de donner la priorité au développement des marchés intérieurs (locaux, nationaux, régionaux) et non à l'exportation;

- de donner la priorité aux attentes de la population en termes d'alimentation, des autres besoins vitaux, de l'environnement, et non au commerce des multinationales;

- d'interdire les brevets sur le vivant ainsi que la production et importation des organismes génétiquement modifiés (OGM).

Le 17 mai 2001. 


\section{Annexe 2: Constitution fédérale}

\section{Art. 104 Agriculture}

1 La Confédération veille à ce que l'agriculture, par une production répondant à la fois aux exigences du développement durable et à celles du marché, contribue substantiellement:

a. à la sécurité de l'approvisionnement de la population;

$b$. à la conservation des ressources naturelles et à l'entretien du paysage rural;

c. à l'occupation décentralisée du territoire.

2 En complément des mesures d'entraide que l'on peut raisonnablement exiger de l'agriculture et en dérogeant, au besoin, au principe de la liberté économique, la Confédération encourage les exploitations paysannes cultivant le sol.

${ }^{3}$ Elle conçoit les mesures de sorte que l'agriculture réponde à ses multiples fonctions. Ses compétences et ses tâches sont notamment les suivantes:

a. elle complète le revenu paysan par des paiements directs aux fins de rémunérer équitablement les prestations fournies, à condition que l'exploitant apporte la preuve qu'il satisfait à des exigences de caractère écologique;

b. elle encourage, au moyen de mesures incitatives présentant un intérêt économique, les formes d'exploitation particulièrement en accord avec la nature et respectueuses de l'environnement et des animaux;

c. elle légifère sur la déclaration de la provenance, de la qualité, des méthodes de production et des procédés de transformation des denrées alimentaires;

d. elle protège l'environnement contre les atteintes liées à l'utilisation abusive d'engrais, de produits chimiques et d'autres matières auxiliaires;

$e$. elle peut encourager la recherche, la vulgarisation et la formation agricoles et octroyer des aides à l'investissement;

f. elle peut légiférer sur la consolidation de la propriété foncière rurale.

${ }^{4}$ Elle engage à ces fins des crédits agricoles à affectation spéciale ainsi que des ressources générales de la Confédération. 